\title{
Can Antibody Galactosylation Be Used to Improve Radioimmunotherapy of Induced Peritoneal Carcinomatosis of Colonic Origin in the Rat?
}

\author{
Frits Aarts, ${ }^{1}$ Thijs Hendriks, ${ }^{1}$ Annemarie Eek, ${ }^{2}$ Wim. J.G. Oyen, ${ }^{2}$ \\ Robert P. Bleichrodt, ${ }^{1}$ and Otto C. Boerman ${ }^{2}$
}

\begin{abstract}
In radioimmunotherapy (RIT), hematologic toxicity is the dose-limiting toxicity due to the long circulatory halflife of the antibody. Although intraperitoneal (i.p.) RIT results in high uptake of i.p. growing tumors, the radiolabeled antibody enters the circulation, resulting in bone marrow toxicity. Carbohydrate modification of antibodies could induce accelerated clearance of the antibody via the hepatic asialoglycoprotein receptor, thereby reducing exposure to normal tissues. In this study, we investigated whether galactosylation of an antibody in a model of peritoneal carcinomatosis (PC) of colonic origin could be used to improve targeting of i.p. growing tumors. Therefore, the biodistribution of the galactosylated and nongalactosylated anti-CC531 antibody, MG1, after i.p. injection was determined in a model of peritoneal carcinomatosis of CC-531 colon tumors in Wag/Rij rats. Uptake of the radiolabeled antibodies in the tumor and relevant organs was determined at 2, 4, 24, and 48 hours after injection. Galactosylation of the antibody did not affect the binding affinity of MG1. Remarkably, the uptake of Gal-MG1 in tumors was higher than that of MG1 at 2 and 4 hours after injection. After 24 and 48 hours, uptake of Gal-MG1 in tumor tissue was lower than that of MG1. Gal-MG1 cleared from the blood within hours after administration. At 2-24 hours after administration, tumor-to-blood ratios obtained with GalMG1 were significantly higher than those obtained with unmodified MG1. Antibody galactosylation resulted in improved tumor-non-tumor ratios after i.p. injection in a model of PC. This could improve the efficiency of RIT, especially in combination with short-lived nonresidualizing radionuclides.
\end{abstract}

Key words: radioimmunotherapy, peritoneal carcinomatosis, galactosylation, cytoreductive surgery

\section{Introduction}

$\mathbf{R}^{2}$ adioimmunotherapy (RIT) comprises the selective irradiation of tumor cells with radiolabeled antitumor antibodies (Abs). RIT has been shown to be an effective treatment in hematologic malignancies. ${ }^{1}$ However, in solid cancers, RIT is less effective, which is partly due to their intrinsic radioresistance and a limited uptake and penetration of Abs in solid tumors. ${ }^{2}$ An inverse relation has been shown to exist between the size of the lesion and the uptake of the radiolabeled antibody. ${ }^{3}$ Therefore, RIT seems to be an attractive adjuvant therapy after surgical debulking procedures, leaving only a microscopic residual tumor.
We have shown previously that the adjuvant intraperitoneal (i.p.) administration of RIT after cytoreductive surgery (CS) is an effective treatment for experimental peritoneal carcinomatosis (PC) of colonic origin. 4,5 Survival in Wag/Rij rats with i.p. CC531 (colon carcinoma) tumors improved significantly when CS was followed by the i.p. administration of RIT with $2 \mathrm{mCi}$ of the ${ }^{177} \mathrm{Lu}$-labeled antiCC531 antibody, MG1. The effect was most explicit when RIT was administered immediately after surgery. In addition, RIT was found to be at least as effective as hyperthermic i.p. chemotherapy (HIPEC), which is the current standard of care, while it resulted in significantly less treatment-related toxicity. ${ }^{6}$

${ }^{1}$ Departments of Surgery and ${ }^{2}$ Nuclear Medicine, Radboud University Nijmegen Medical Centre, Nijmegen, The Netherlands

Address correspondence to: Frits Aarts; Department of Surgery, Radboud University Nijmegen Medical Centre, Nijmegen; P.O. Box 9101, 6500 HB Nijmegen, The Netherlands; Tel.: +31-24-3615333; Fax: +31-24-3540501

E-mail: F.Aarts@chir.umcn.nl 
In order to enhance the therapeutic efficacy of radiolabeled Abs, different strategies have been pursued, ranging from locoregional administration of these Abs, as described above, to the application of $\mathrm{Ab}$ fragments and pretargeting systems. ${ }^{7}$ We studied galactosylation of the antitumor antibody as a new method to enhance the efficacy of i.p.-applied RIT for the treatment of i.p. tumors. After direct tumor targeting following i.p. administration, this modification induces very rapid blood clearance via the hepatic asialoglycoprotein receptor (AGPR). This could result in high tumor-to-blood and tumor-to-non-tumor ratios. 8,9 As a consequence of rapid clearance, the radiation dose that is delivered to the bone marrow will be reduced, thus lowering hematologic toxicity. Ultimately, this effect would allow the administration of higher doses of radioactivity and potentially increase the efficacy of RIT. To test this hypothesis, the tumor targeting and uptake in nontarget tissues of i.p.-injected Gal-MG1 was compared with that of nongalactosylated MG1 in Wag/Rij rats with small-volume peritoneal CC-531 carcinomatosis.

\section{Materials and Methods}

\section{Reagents}

Antibody. The murine MG1 monoclonal antibody (mAb), an anti-CC531 IgG2a mAb (Antibodies for Research Applications BV, Gouda, The Netherlands) specifically directed against an 80-kDa cell-surface antigen expressed on CC531 cells, was used in these studies. The MG1 mAb localizes preferentially in tumors when injected in rats bearing CC531 tumors. ${ }^{10}$

Galactosylation. To galactosylate MG1, cyanomethyl2,3,4,6-tetra-O-acetyl-1-thio-beta-D-galactopyranoside (CAGP, C-4141; Sigma-Aldrich Chemie B.V., Zwijndrecht, The Netherlands) was dissolved in methanol at a concentration of $34 \mathrm{mg} / \mathrm{mL}$ and mixed with an 0.1 volume of $0.1 \mathrm{M}$ of sodium methoxide, also in methanol. After 48 hours at room temperature, the methanol was evaporated (Argon flow, $35^{\circ} \mathrm{C}$ ) to dryness and the residue was dissolved in $1 \mathrm{~mL}$ of $0.025 \mathrm{M}$ sodium borate buffer ( $\mathrm{pH} 8.5$ ), containing $5 \mathrm{mg}$ of MG1. After 2 hours at room temperature, the reaction mixture was dialyzed against phosphate-buffered saline (PBS).

To determine the number of galactosyl groups that were conjugated per MG1 molecule, the method described by Dubois et al. was used, with minor modifications. ${ }^{11}$ To 0.1 $\mathrm{mL}$ of galactose solution $(0.8-20 \mu \mathrm{g}), 0.1 \mathrm{~mL}$ of $5 \%$ phenol solution was added and mixed. Then, $0.5 \mathrm{~mL}$ of concentrated $\mathrm{H}_{2} \mathrm{SO}_{4}$ was added to the solution. The mixture was vortexed and allowed to stand for 30 minutes at room temperature. The galactosylated antibody solution $(0.1 \mathrm{~mL})$ was similarly treated. Absorbance at $490 \mathrm{~nm}$ was measured with an Amersham Pharmacia BioTech Benelux (Zwijndrecht, The Netherlands) Ultrospec 2000 spectrophotometer. It was determined that 25 molecules of galactose were conjugated per MG1 molecule.

Radioiodination. Galactosylated MG1 was radioiodinated with ${ }^{125} \mathrm{I}$ (Amersham, Den Bosch, The Netherlands), while the ungalactosylated MG1 was radioiodinated with ${ }^{131} \mathrm{I}$, using the iodogen method, as described previously. ${ }^{12}$ Briefly, the antibody $(1 \mathrm{mg})$ and the radioiodide $(600 \mu \mathrm{Ci})$ were incubated at room temperature in PBS (pH 7.4) in an Eppen-

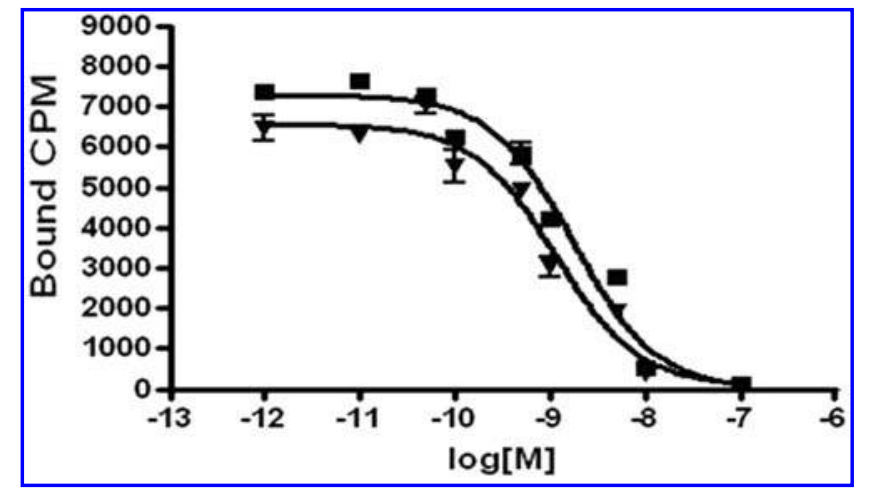

FIG. 1. Competitive binding assay showing the affinity of both galactosylated and ungalactosylated MG1 for CC531 cells. $\mathbf{\square}, \mathrm{MG1} ; \boldsymbol{\nabla}, \mathrm{Gal}-\mathrm{MG} 1$.

dorf tube (Omnilabo, Breda, The Netherlands), coated with $50 \mu \mathrm{g}$ of iodogen. After 10 minutes, the reaction was stopped by adding $100 \mu \mathrm{L}$ of a saturated tyrosine solution. The radioiodinated antibodies were purified on a PD-10 column (Amersham Biosciences, Uppsala, Sweden), eluted with PBS and $0.5 \%$ bovine serum albumin (BSA). This resulted in a specific activity of $0.56 \mu \mathrm{Ci} / \mu \mathrm{g}$ for MG1 and $0.48 \mu \mathrm{Ci} / \mu \mathrm{g}$ for galactosylated MG1.

Competitive binding assay. To determine the effect of galactosylation on the affinity of the MG1 antibody, the $\mathrm{IC}_{50}$ value of Gal-MG1 and MG1 was determined in a competitive binding assay. Binding of the ${ }^{125} \mathrm{I}$-labeled MG1 was competed by unlabeled nongalactosylated MG1 or Gal-MG1 and in a concentration-dependent manner. ${ }^{125}$ I-labeled MG1 was used as the tracer in this assay. Six-well Costar culture plates (Corning Life Sciences B.V., Schiphol-Rijk, The Netherlands) were seeded with CC531 cells and cultured until confluency. The plates were washed twice with PBS. Then, $3 \mathrm{~mL}$ of binding buffer containing 50,000 cpm of ${ }^{125} \mathrm{I}-\mathrm{MG} 1$ with a serial dilution $\left(0 \mathrm{mg} / \mathrm{mL}\right.$ to $\left.1.5 \times 10^{-7} \mathrm{mg} / \mathrm{mL}\right)$ of nonlabeled MG1 or galactosylated MG1 in binding buffer was incubated in the wells at $37^{\circ} \mathrm{C}$ for 1 hour. After incubation, the plates were washed three times with PBS. Radioactivity in each well was determined in a $\gamma$-counter (1480 Wizard; Wallac, Turku, Finland). $\mathrm{IC}_{50}$ values of MG1 and Gal-MG1 were calculated by nonlinear regression, using GraphPad Prism 4.0 (GraphPad Prism Software, San Diego, CA).

\section{Model of peritoneal carcinomatosis}

The syngeneic rat colon carcinoma cell line, CC531, originally induced in Wag/Rij rats by the intravenous injection of 1,2-dimethylhydrazine, ${ }^{13}$ was cultured and maintained as a monolayer in RPMI-1640 medium (GIBCO, BRL Life Sciences Technologies, The Netherlands), supplemented with $10 \%$ fetal calf serum (FCS; GIBCO), 2 mM L-glutamine, penicillin $[100 \mathrm{U} / \mathrm{mL}]$, and streptomycin $[100 \mu \mathrm{g} / \mathrm{mL}]$ at $37^{\circ} \mathrm{C}$ in a humidified atmosphere with $5 \% \mathrm{CO}_{2}$. Tumor cells were harvested from culture flasks with $0.25 \%$ trypsin and resuspended in RPMI-1640 medium to a concentration of $1 \times 10^{6}$ cells/mL. Two (2) $\mathrm{mL}$ of this cell suspension was injected i.p., as previously described. ${ }^{14}$ Male WAG/Rij rats (10-12 weeks old, body weight 240-260 g; Harlan Horst, The Netherlands) were housed under nonsterile standard condi- 


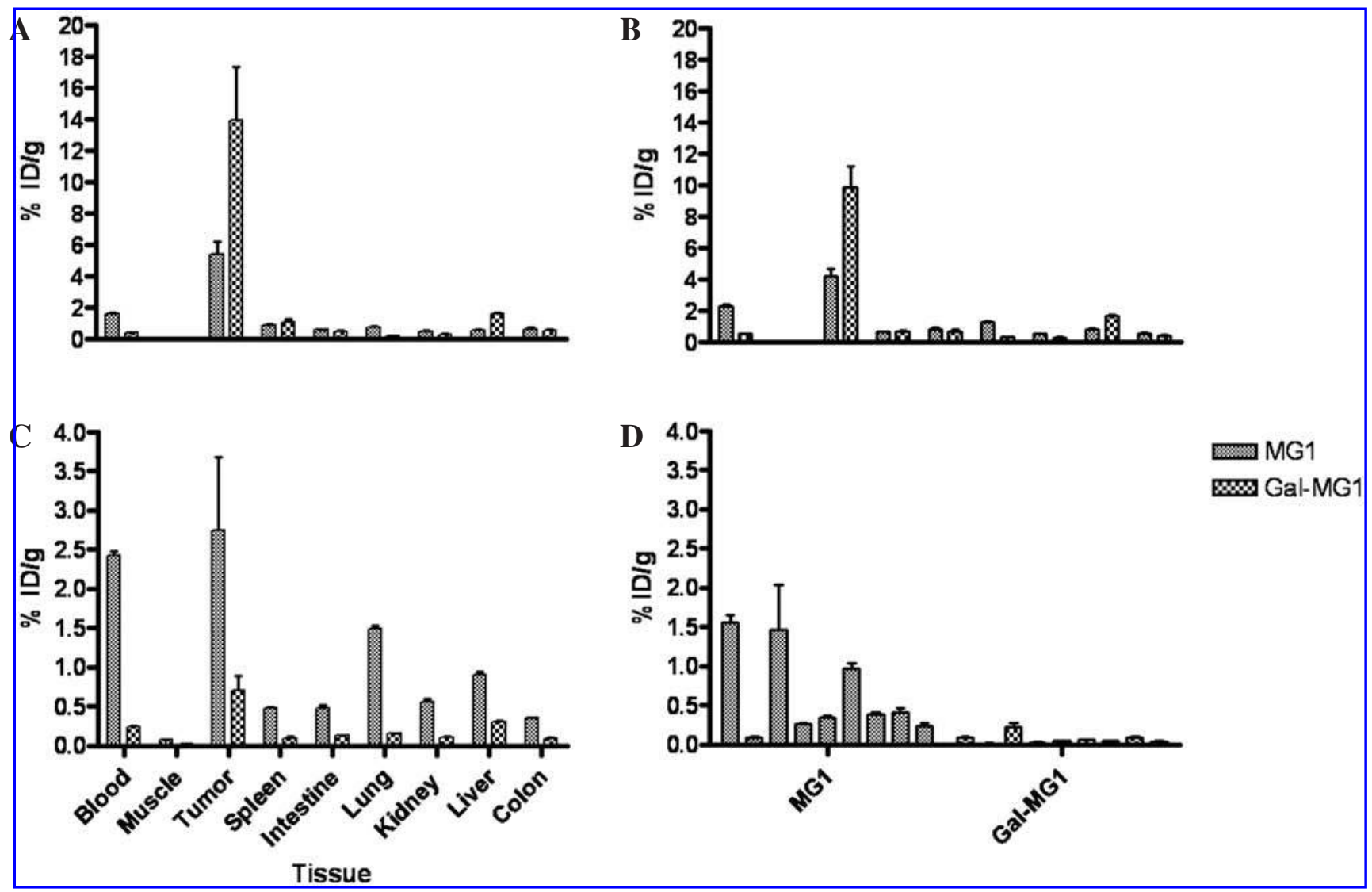

FIG. 2. Biodistribution results of Gal-MG1 and MG1 at (A) 2, (B) 4, (C) 24, and (D) 48 hours after intraperitoneal administration, expressed as the mean percent injected dose per gram of tissue. Bars indicate the standard error of the mean. MG1; * Gal-MG1.

tions (temperature, $20-24^{\circ} \mathrm{C}$; relative humidity, $50 \%-60 \%$; 12-hours light and dark cycle) in filter-topped cages (2-3 rats per cage) with free access to food (Ssniff; Bio Services Uden, The Netherlands) and water. Rats were accustomed to laboratory conditions for at least 1 week before experimental use. All experiments were approved by the local Animal Welfare Committee of Radboud University (Nijmegen, The Netherlands) and were carried out in accordance with the Dutch Animal Welfare Act of 1997.

\section{Biodistribution}

First, the optimal CAGP:IgG conjugation ratio to prepare the Gal-MAb conjugates was determined by using the unrelated anti-CAIX MAb, G250. G250-Gal conjugates were prepared at four different molar CAGP:IgG conjugation ratios (60:1 to 3000:1). The four Gal-G250 conjugates were labeled with I-125 and injected into rats via the tail vein. All GalG250 conjugates cleared rapidly from the (blood level $<0.7 \%$ injected dose per gram [ID/g] at 5 minutes postinjection). Based on these studies, the lowest conjugation ratio (1:60) was used to prepare the Gal-MG1 conjugate in the biodistribution studies.

The biodistribution of galactosylated MG1 labeled with ${ }^{125} \mathrm{I}$ and nongalactosylated MG1 labeled with ${ }^{131} \mathrm{I}$ in Wag/Rij rats with i.p. growing CC531 tumor nodules was determined at 2, 4, 24, and 24 hours ( $n=5$ /group) after i.p. injection. Both radiolabeled Abs $\left(1 \mathrm{~mL} / \mathrm{rat}, 8 \mu \mathrm{Ci}{ }^{131} \mathrm{I}-\mathrm{MG} 1 / \mathrm{rat}, 9 \mu \mathrm{Ci}\right.$
${ }^{125}$ I-Gal-MG1/rat) were administered simultaneously. At dissection, samples of the tumor, blood, liver, spleen, kidneys, intestine, lung, and muscle were removed and immediately weighed. Radioactivity was measured in a well-type $\gamma$-counter (Wizard; Pharmacia-LKB). To correct for physical decay and to calculate the uptake of the radiolabeled antibody in each sample as a fraction of the injected dose, aliquots of the injected dose were counted simultaneously. The uptake was expressed as the $\% \mathrm{ID} / \mathrm{g}$.

\section{Statistical analysis}

Statistical analysis was performed by using SPSS (SPSS Inc., Chicago, IL) software and Graphpad Prism, version 4.0 (Graphpad Software Inc., San Diego CA) for analysis. For single comparison, the paired $t$-test was used. $\mathrm{IC}_{50}$ values of the MG1 were calculated by nonlinear regression, using GraphPad Prism (GraphPad Software).

\section{Results}

\section{Reagents}

Affinity. The affinity of the galactosylated MG1 was determined in a competitive binding assay. The results are shown in Figure 1. Binding of ${ }^{125} \mathrm{I}-\mathrm{MG} 1$ to CC531 cells was competed for by both Gal-MG1 and MG1 in a concentration-dependent manner. Both $\mathrm{IC}_{50}$ values were in the nanomolar range, with $1.6 \mathrm{nM}$ for MG1 and $1.2 \mathrm{nM}$ for Gal-MG1. 


\section{Biodistribution}

The results of the biodistribution studies are summarized in Figure 2A-2D. There was a preferential uptake of both radiolabeled antibody preparations in the i.p. tumors. At 2 and 4 hours postinjection, the uptake of Gal-MG1 in tumor tissue $(14.0 \pm 7.5$ and $9.9 \pm 3.0 \% \mathrm{ID} / \mathrm{g}$, respectively) was significantly higher than that of the ungalactosylated MG1 $(5.4 \pm 1.9$ and $4.3 \pm 1.0 \% \mathrm{ID} / \mathrm{g} ; p<0.04)$. Tumor-to-blood ratios at 2 and 4 hours after injection for Gal-MG1 were 10fold higher than those obtained with ungalactosylated MG1 (32.4 \pm 18.9 vs. $3.2 \pm 1.48$ at 2 hours and $18.7 \pm 5.51$ vs. $1.9 \pm$ 0.59 at 4 hours; $p<0.0001)$. At 24 and 48 hours after administration, the uptake of Gal-MG1 in tumor tissue $(0.7 \pm$ $0.2 \% \mathrm{ID} / \mathrm{g}$ at 24 hours and $0.2 \pm 0.1 \% \mathrm{ID} / \mathrm{g}$ at 48 hours) tended to be lower than that of ungalactosylated MG1 (2.6 \pm $1.0 \% \mathrm{ID} / \mathrm{g}, p=0.06$, and $1.5 \pm 1.3 \% \mathrm{ID} / \mathrm{g}, p=0.10$, respectively). Although the tumor uptake of the galactosylated $\mathrm{Ab}$ was lower at 24 and 48 hours after administration, the tumor-to-blood ratios for Gal-MG1 remained significantly higher at 24 hours $(2.70 \pm 0.81)$ than those obtained with ungalactosylated MG1 $(1.04 \pm 0.39 ; p<0.04)$. After 48 hours, there was no significant difference in tumor-to-blood ratios. In addition, uptake of Gal-MG1 in all normal tissues, except the liver, was lower than that of MG1 at 2 and 4 hours after injection. One (1) and 2 days after administration, liver uptake was lower for Gal-MG1, as compared to MG1 (Figure 2C and 2D). The uptake in nontarget organs, such as muscle, lung, spleen, and kidneys, was low for both Abs, albeit lower for Gal-MG1 after 24 and 48 hours.

The total area under the curve (AUC) for Gal-MG1 for the tumor was $192 \% \mathrm{ID} / \mathrm{g} \cdot \mathrm{h}$, as compared to $90 \% \mathrm{ID} / \mathrm{g} \cdot \mathrm{h}$ for ungalactosylated MG1. For the blood, the total AUC for the galactosylated $\mathrm{Ab}$ was $7.9 \% \mathrm{ID} / \mathrm{g} \cdot \mathrm{h}$, as compared to 24.5 $\% \mathrm{ID} / \mathrm{g} \cdot \mathrm{h}$ for MG1. The ratio of total AUC of Gal-MG1 in the tumor/AUC for blood was 24.3, as compared to 3.7 for the ungalactosylated MG1.

\section{Discussion}

The aim of this study was to investigate whether galactosylation of antitumor antibody MG1 could improve the preferential targeting of i.p. tumors in a rat model. The rationale for using a carbohydrated antitumor antibody is that galactosylated antibodies clear very rapidly from the blood via the hepatic asialoglycoprotein receptor (AGPR), resulting in low blood levels. ${ }^{8,9}$ In cases of i.p. tumors, this could result in optimal tumor targeting after i.p. administration with concomitant rapid clearance when the antibody enters the circulation.

Indeed, at all the time points after i.p. injection, blood levels of Gal-MG1 were significantly lower than the blood levels of MG1. Ong et al. investigated the administration of galactosylated Abs in a model of an i.p. ovarian cancer cell line. ${ }^{15}$ The investigators described a tumor uptake of 4.3 $\%$ ID/g 28 hours after injection with a peritoneal retention of the administered $\mathrm{Ab}$ of $10 \% 24$ hours after administration. In these experiments, however, peritoneal clearance was disturbed by the application by Freund's adjuvant, causing a major inflammatory response, thus not resembling normal physiology. Sharma et al. used a model of subcutaneously growing human colon cancer xenografts in mice. ${ }^{16}$ The investigators used blocking agents to obtain a prolonged circulation of the galactosylated antibodies for the duration of 8 hours. This resulted in tumor-to-blood ratios of 45:1.
Galactosylation of MG1 did not affect the affinity of the $\mathrm{Ab}$ for the MG1 antigen. Remarkably, within the first 24 hours after administration, tumor uptake of the Gal-Ab was significantly higher (2.6-fold at 2 hours and 2.3-fold at 4 hours after administration) than that of the nongalactosylated antibody. The higher tumor uptake could be due to a longer i.p. retention time of the Gal-Ab. The more negative charge of the Gal-MG1 could result in a slower transit from the i.p. cavity to the circulation. The lower tumor uptake of the galactosylated MG1 after 24 and 48 hours is due to the reduced blood levels of Gal-MG1. We assumed that antibody uptake in i.p. tumors after i.p. injection was the result of delivery, both directly from the peritoneal cavity as well as via the i.v. route. After 24 hours, most of the galactosylated Abs had cleared from the peritoneal cavity. As a consequence of galactosylation, the antibody would have cleared from the circulation, whereas the ungalactoslated antibody would still circulate at relatively high levels in the blood. ${ }^{17-19}$

Despite the significantly lower tumor uptake of Gal-MG1 after 24 and 48 hours, the total AUC of the tumor was higher for the galactosylated antibody. Moreover, the ratio of $\mathrm{AUC}_{\text {(tumor) }} / \mathrm{AUC}_{\text {(blood) }}$ for Gal-MG1 was 7 times higher than the ratio for ungalactosylated MG1. Thus, when normalized for the AUC of the blood, the $\mathrm{AUC}_{\text {tumor }}$ of the galactosylated $\mathrm{Ab}$ was 7 times higher. Bone marrow toxicity is related to the $\mathrm{AUC}_{\text {blood }}$. The $\mathrm{AUC}_{\text {tumor }}$ can be normalized for $\mathrm{AUC}_{\mathrm{blood}}$, and thus the comparison for both AUC for tumor at an equitoxic bone marrow dose can be determined. This would indicate that the radiation dose of ${ }^{131} \mathrm{I}-\mathrm{Gal}-\mathrm{MG} 1$ to the tumor is approximately 7 times higher than that of ${ }^{131} \mathrm{I}-\mathrm{MG} 1$ at an equitoxic dose. Considering the high initial tumor uptake, the high tumor-to-non-tumor ratios after i.p. injection of Gal$\mathrm{Ab}$ and the favorable normalized $\mathrm{AUC}_{\text {tumor }}$, RIT, using these modified Abs, seems feasible. In this model, tumor uptake peaks within 24 hours after i.p. administration. As a result, the favorable effect of the galactosylated $\mathrm{Ab}$ could be more pronounced when other radionuclides with relatively short half-lives and nonradiometals were used. In addition, since clearance of galactosylated antibodies is via the hepatic AGPR, the use of residualizing radiometals, such as ${ }^{177} \mathrm{Lu}$ and ${ }^{90} \mathrm{Y}$, could result in increased radiation dose to the liver. Almqvist et al. showed excellent tumor targeting of subcutaneous colon tumors in a mouse model with a low liver uptake of the ${ }^{211}$ At-labeled antibody A33. ${ }^{20}{ }^{211}$ At is a nonresidualizing radionuclide with a relatively short half-life (7.2 hours) and has exquisite characters for RIT of i.p. tumors with galactosylated antibodies.

\section{Conclusions}

Therefore, the i.p. application of RIT for the treatment of peritoneal carcinomatosis, using galactosylated antibodies, needs further exploration.

\section{Disclosure Statement}

No competing financial interests exist.

\section{References}

1. Fisher RI, Kaminski MS, Wahl RL, et al. Tositumomab and iodine-131 tositumomab produces durable complete remis- 
sions in a subset of heavily pretreated patients with lowgrade and transformed non-Hodgkin's lymphomas. L Clin Oncol 2005;23:7565.

2. Heldin $\mathrm{CH}$. High interstitial fluid pressure-an obstacle in cancer therapy. Nat Rev Cancer 2004;4:806.

3. Behr TM, Sharkey RM, Juweid ME, et al. Variables influencing tumor dosimetry in radioimmunotherapy of CEA-expressing cancers with anti-CEA and antimucin monoclonal antibodies. I Nucl Med 1997;38:409.

4. Koppe MJ, Hendriks T, Boerman OC, et al. Radioimmunotherapy is an effective adjuvant treatment modality after cytoreductive surgery of peritoneal carcinomatosis of colonic origin. L Nucl Med 2006;47:1867.

5. Aarts F, Koppe MJ, Hendriks T, et al. Timing of adjuvant radioimmunotherapy after cytoreductive surgery in experimental peritoneal carcinomatosis of colorectal origin. Ann Surg Oncol 2007;14:533.

6. Aarts F, Hendriks T, Boerman OC, et al. A comparison between radioimmunotherapy and hyperthermic intraperitoneal chemotherapy for the treatment of peritoneal carcinomatosis of colonic origin in rats. Ann Surg Oncol 2007;14: 3274.

7. Boerman OC, van Schaijk FG, Oyen WJ, et al. Pretargeted radioimmunotherapy of cancer: Progress step by step. $[\mathrm{Nucl}$ Med 2003;44:400.

8. Ashwell G, Harford J. Carbohydrate-specific receptors of the liver. Annu Rev Biochem 1982;51:531.

9. Morell AG, Gregoriadis G, Scheinberg IH, et al. The role of sialic acid in determining the survival of glycoproteins in the circulation. L Biol Chem 1971;246:1461.

10. Hagenaars M, Koelemij R, Ensink NG, et al. The development of novel mouse monoclonal antibodies against the CC531 rat colon adenocarcinoma. Clin Exp Metast 2000; 18:281.
11. Dubois M, Gilles KA, Hamilton JK. Colorimetric method for determination of sugars and related substances. $\underline{\text { Annal Chem }}$ 1956;28:350.

12. Fraker PJ, Speck JC, Jr. Protein and cell membrane iodinations with a sparingly soluble chloroamide, 1,3,4,6-tetrachloro-3a,6a-diphrenylglycoluril. Biochem Biophys Res Commun 1978;80:849.

13. Zedeck MS. A model system for studies of colon carcinogenesis: Tumor induction by a single injection of methylazoxymethanol acetate. L Natl Cancer Inst 1974;53:1419.

14. Lopes Cardozo AM, Gupta A, Koppe MJ, et al. Metastatic pattern of CC531 colon carcinoma cells in the abdominal cavity: An experimental model of peritoneal carcinomatosis in rats. Eur J Surg Oncol 2001;27:359.

15. Ong GL, Ettenson D, Sharkey RM, et al. Galactose-conjugated antibodies in cancer therapy: Properties and principles of action. Cancer Res 1991;51:1619.

16. Sharma SK, Bagshawe KD, Burke PJ, et al. Galactosylated antibodies and antibody-enzyme conjugates in antibody-directed enzyme prodrug therapy. Cancer 1994;73:1114.

17. Colcher D, Esteban J, Carrasquillo JA, et al. Complementation of intracavitary and intravenous administration of a monoclonal antibody (B72.3) in patients with carcinoma. Cancer Res 1987;47:4218.

18. Koppe MJ, Soede AC, Pels W, et al. Experimental radioimmunotherapy of small peritoneal metastases of colorectal origin. Int J Cancer 2003;106:965.

19. van Zanten-Przybysz I, Molthoff CF, Roos JC, et al. Influence of the route of administration on targeting of ovarian cancer with the chimeric monoclonal antibody MOv18: i.v. vs. i.p. Int J Cancer 2001;92:106.

20. Almqvist $Y$, Steffen AC, Lundqvist $H$, et al. Biodistribution of ${ }^{211}$ At-labeled humanized monoclonal antibody A33. Cancer Biother Radiopharm 2007;22:480. 

This article has been cited by:

1. Christof Seidl, Christine Zöckler, Roswitha Beck, Leticia Quintanilla-Martinez, Frank Bruchertseifer, Reingard Senekowitsch-Schmidtke. 2011. 177Lu-immunotherapy of experimental peritoneal carcinomatosis shows comparable effectiveness to 213Bi-immunotherapy, but causes toxicity not observed with 213Bi. European Journal of Nuclear Medicine and Molecular Imaging 38:2, 312-322. [CrossRef] 